Rot or speed control of doubl y fed i nduct i on gener at or wi nd turbi nes usi ng adapt i ve maxi mum power poi nt tracking

\begin{tabular}{|l|l|}
\hline 著者 & Phan Di nh- Chung, Yananot o Shi ger u \\
\hline 著者別表示 & 山本 茂 \\
\hline $\begin{array}{l}\text { j our nal or } \\
\text { publ i cat i on ti t l e }\end{array}$ & Energy \\
\hline vol une & 111 \\
\hline page range & $377-388$ \\
\hline year & 2016-09-15 \\
\hline URL & ht t p: //doi . or g/10. 24517/00009543 \\
\hline
\end{tabular}




\title{
Rotor Speed Control of Doubly Fed Induction Generator Wind Turbines using Adaptive Maximum Power Point Tracking
}

\author{
Dinh-Chung Phan ${ }^{\mathrm{a}, \mathrm{b}}$, Shigeru Yamamoto ${ }^{\mathrm{c}, *}$ \\ ${ }^{a}$ Faculty of Electrical Engineering, Danang University of Science and Technology, 54-Nguyen Luong Bang, Danang 550000, Vietnam \\ ${ }^{b}$ Division of Electrical Engineering and Computer Science, Graduate School of Natural Science and Technology, Kanazawa University, Kakuma, \\ Kanazawa, Ishikawa 920-1192, Japan \\ ${ }^{c}$ Faculty of Electrical and Computer Engineering, Institute of Science and Engineering, Kanazawa University, Kakuma, Kanazawa, Ishikawa \\ 920-1192, Japan
}

\begin{abstract}
This paper proposes a new method for obtaining the maximum power output of a doubly fed induction generator (DFIG) wind turbine. This scheme does not require the precise parameters of the wind turbine or any information about the wind speed or wind sensor. The maximum power point tracking (MPPT) ability of the proposed method is theoretically proven under some certain assumptions. To obtain the required control performance, several control parameters may be adopted. Particularly, the control method is constructed on the basis of the Lyapunov function. The quality of the proposed method is verified by the numerical simulation of a 1.5-MW DFIG wind turbine. The simulation results show that the wind turbine implemented with the proposed method can track the optimal operation point. Furthermore, the energy output of the DFIG wind turbine using the proposed method is higher compared to conventional methods under the same conditions.

Keywords: doubly fed induction generator, Lyapunov function, maximum power point tracking, maximum energy, adaptive control
\end{abstract}

\section{Introduction}

To optimally utilize wind energy, the energy conversion efficiency of wind turbines must reach the utmost limit. Therefore, maximum power point tracking (MPPT) is an essential target in wind turbine control. To track the maximum power point, the rotor speed of the wind turbine/generator should be adjustable. Hence, the concept of a variable-speed wind turbine (VSWT) was proposed. According to [1-5], when the generator in a VSWT operates at variable speeds, its output is often synchronized with the grid via a converter system. Depending on the type of generator used in the VSWT, the converter's size will vary [1]. With VSWTs based on synchronous generators, permanent magnetic synchronous generators (PMSGs), or squirrel-cage induction generators, they require a full converter on the generator's stator side [3-5]. However, for VSWTs that use a doubly fed induction generator (DFIG), a partial

\footnotetext{
${ }^{*}$ Corresponding author

Email addresses: chungpy99d6@yahoo.com (Dinh-Chung Phan), shigeru@se.kanazawa-u.ac.jp (Shigeru Yamamoto)
} 
converter is required on the rotor side [2]. In other words, compared to a full converter-based VSWT, the use of a DFIG wind turbine is more economical; in fact, DFIG wind turbines are more frequently used in large wind farms. Therefore, control for a MPPT target in DFIG-based wind turbines has become an interesting topic.

To track the maximum power point during operation, a wind turbine must be generally equipped with a good controller integrated with a comprehensive MPPT algorithm. Many MPPT methods have been proposed [5-13]. Original methods are based on the characteristic curve. They use the curve of the optimal power versus wind speed, for example, or the optimal tip-speed ratio of a wind turbine and wind data to determine the reference signal for the controller [6, 8]. These methods are called wind-data-based methods. Generally, with wind-data-based methods, the MPPT ability of a wind turbine is appreciably high if accurate wind data is available. However, because of the rapid natural fluctuation of wind, wind speed measurement is hardly reliable [14]. To overcome this drawback, other methods such as the MPPT-curve method [10-13] and perturbation and observation $(\mathrm{P} \& \mathrm{O})$ method [7] were suggested. They operate basically on the output of the generator; hence, they are called wind speed-sensorless methods. Compared to the wind-data-based methods, the wind speed-sensorless methods cannot track the optimum point as efficiently as [15]. However, this method is often implemented in wind turbines because there is no requirement for an anemometer. The $\mathrm{P} \& \mathrm{O}$ method is originally applied for extremum seeking in small inertia systems such as photovoltaic power systems or small-size PMSG wind turbines with a DC/DC converter [5, 7]. Unlike the P\&O method, the MPPT-curve method, which indexes the current power output (or rotor speed) as well as the wind turbine's MPPT curve to determine the reference rotor speed (or power output) [11-13], can apply to both large- and-small scale wind turbines; it is more efficient and does not require any perturbation signal [8]. However, for the high inertia of a generator wind turbine system, a wind turbine using the MPPT-curve method cannot track the maximum point as rapidly as a wind turbine using the wind-data-based method [15]. In terms of designing the controller for a wind turbine, traditional proportional-integral (PI) control is used for many purposes, including rotor-speed, current, and power control [11, 12]. A drawback of PI control is that stability is not theoretically guaranteed [1, 16, 17]. Thus, sliding-mode control has been recently developed [18-22]. In fact, sliding-mode control has been applied to the rotor speed [20-22]. However, wind speed measurement is prerequisite for sliding mode control. In [1], the authors improved the MPPT-curve method, which is only applied to power control on the rotor-side converter. Hence, an improvement of the MPPT-curve method integrated with a controller, which is designed basically on the Lyapunov control theory, to adjust the rotor speed without an available wind sensor is currently anticipated.

This research suggests a new scheme to maximize the energy output of a DFIG wind turbine without any information about the wind data or an available anemometer. The proposed scheme is based on the improvement of the wind turbine's MPPT curve. A new controller based on Lyapunov control theory will be designed for rotor speed adjustment purposes. The efficiency of the proposed scheme will be verified, analyzed, and compared with the conventional MPPT curve method with PI controllers by the simulation of a 1.5-MW DFIG wind turbine in a MATLAB/Simulink environment. 


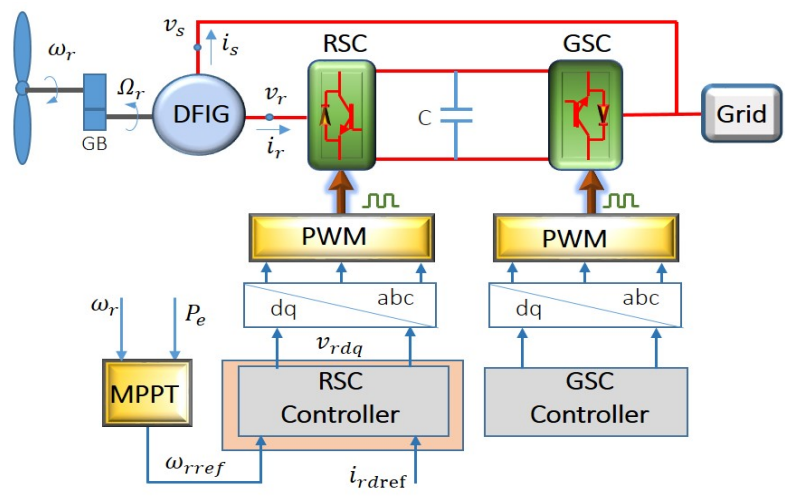

Fig. 1: Overall system of the DFIG wind turbine

\section{DFIG wind turbine}

A DFIG wind turbine [1, 23] consists of a wind turbine and doubly fed induction generator (DFIG). The wind turbine is linked to the generator through a gearbox and shaft system, as shown in Fig. 1 The stator side of the DFIG is often connected directly to the grid, whereas the rotor side is connected through a partial converter, including a rotor-side converter (RSC) and a grid-side converter (GSC).

\subsection{Wind turbine}

Generally, the dynamic equation for a generator-wind turbine system [18] is used to described the rotor speed $\omega_{r}$, mechanical torque $T_{m}$, and electrical torque $T_{e}$ as

$$
J \frac{d}{d t} \omega_{r}(t)=T_{m}(t)-T_{e}(t)
$$

where $J$ is the inertia of the generator-wind turbine system. Moreover, to use the mechanical and electrical power $P_{m}$ and $P_{e}$, respectively, we can rewrite (1) as

$$
J \omega_{r}(t) \frac{\mathrm{d}}{\mathrm{d} t} \omega_{r}(t)=P_{m}(t)-P_{e}(t)
$$

$P_{m}$, which is a function of the tip-speed ratio $\lambda$ and wind speed $V_{w}$, is written as

$$
P_{m}\left(\lambda, V_{w}\right) \triangleq \frac{1}{2} \rho \pi R^{2} C_{p}(\lambda) V_{w}^{3}
$$

where $R, \rho$, and $C_{p}$ are the blade size, air density, and power coefficient, respectively. The power coefficient $C_{p}$ generally depends on the pitch angle $\beta$. Throughout this paper, we fix $\beta$ as a constant. Hence, we simply denote it as $C_{p}(\lambda)$

The tip-speed ratio is

$$
\lambda\left(\omega_{r}, V_{w}\right) \triangleq \frac{R \omega_{r}}{V_{w}}
$$

Hence, we can regard $P_{m}$ as

$$
P_{m}\left(\omega_{r}, V_{w}\right)=\frac{1}{2} \rho \pi R^{2} C_{p}\left(\lambda\left(\omega_{r}, V_{w}\right)\right) V_{w}^{3} .
$$




\section{2. $D F I G$}

The DFIG can be described in the dq frame [1, 24, 25] as

$$
\left\{\begin{aligned}
L_{s} \frac{\mathrm{d}}{\mathrm{d} t} \mathbf{i}_{s}(t) & +L_{m} \frac{\mathrm{d}}{\mathrm{d} t} \mathbf{i}_{r}(t)+R_{s} \mathbf{i}_{s}(t)+\omega_{s} \Theta\left(L_{s} \mathbf{i}_{s}(t)+L_{m} \mathbf{i}_{r}(t)\right) \\
= & \mathbf{v}_{s}(t) \\
L_{r} \frac{\mathrm{d}}{\mathrm{d} t} \mathbf{i}_{r}(t) & +L_{m} \frac{\mathrm{d}}{\mathrm{d} t} \mathbf{i}_{s}(t)+R_{r} \mathbf{i}_{r}(t)+s(t) \omega_{s} \Theta\left(L_{m} \mathbf{i}_{s}(t)+L_{r} \mathbf{i}_{r}(t)\right) \\
= & \mathbf{v}_{r}(t)
\end{aligned}\right.
$$

where $\mathbf{v}_{s} \triangleq\left[\begin{array}{ll}v_{s d} & v_{s q}\end{array}\right]^{\top}, \mathbf{v}_{r} \triangleq\left[\begin{array}{ll}v_{r d} & v_{r q}\end{array}\right]^{\top}, \mathbf{i}_{s} \triangleq\left[\begin{array}{ll}i_{s d} & i_{s q}\end{array}\right]^{\top}$, and $\mathbf{i}_{r} \triangleq\left[\begin{array}{ll}i_{r d} & i_{r q}\end{array}\right]^{\top}$ are the stator-side, rotor-side voltage, stator-side current, and rotor-side current, respectively; $\boldsymbol{\Theta} \triangleq\left[\begin{array}{cc}0 & -1 \\ 1 & 0\end{array}\right]$; and $\omega, R, L$, and $s$ represent the rotational speed, resistance, inductance, and rotor slip, respectively. The subscripts $r, s$, and $m$ stand for rotor side, stator side, and magnetization, respectively. Normally, $\omega_{s}$ is assumed constant. The rotor slip of the DFIG is written as

$$
s(t) \triangleq 1-\frac{p_{n} \Omega_{r}(t)}{\omega_{s}}=1-\frac{p_{n} N \omega_{r}(t)}{\omega_{s}},
$$

where $p_{n}$ is the number of pole pairs, and $N$ is the gearbox ratio defined as the ratio between the rotational speeds of the low-speed shaft $\omega_{r}$ and high-speed shaft $\Omega_{r}$

$$
\Omega_{r}(t) \triangleq N \omega_{r}(t)
$$

Assumption 1. The stator flux is constant and the d-axis of the dq-frame is oriented with the stator flux vector [1]. Hence,

$$
\boldsymbol{\Psi}_{s}(t)=\left[\begin{array}{c}
\Psi_{s d}(t) \\
\Psi_{s q}(t)
\end{array}\right] \equiv\left[\begin{array}{c}
\Psi_{s d} \\
0
\end{array}\right]=L_{s} \mathbf{i}_{s}(t)+L_{m} \mathbf{i}_{r}(t) .
$$

Then,

$$
L_{s} \frac{\mathrm{d}}{\mathrm{d} t} \mathbf{i}_{s}(t)+L_{m} \frac{\mathrm{d}}{\mathrm{d} t} \mathbf{i}_{r}(t)=0
$$

Moreover, the resistance of the stator winding of the generator is zero, i.e., $R_{s}=0$ [1].

Lemma 1. Under Assumption[1] in a DFIG [6, the stator-side voltage becomes constant as

$$
\mathbf{v}_{s}(t)=\left[\begin{array}{ll}
0 & V_{s}
\end{array}\right]^{\top},
$$

where $V_{s}$ is the magnitude of the stator voltage $\left\|\mathbf{v}_{s}(t)\right\|$. Moreover, the rotor-side current $\mathbf{i}_{r}$ and voltage $\mathbf{v}_{r}$ satisfy

$$
\sigma \frac{\mathrm{d}}{\mathrm{d} t} \mathbf{i}_{r}(t)=\mathbf{A}_{r}(t) \mathbf{i}_{r}(t)-\mathbf{v}_{r}(t)+\mathbf{d}(t),
$$


where

$$
\begin{aligned}
& \sigma \triangleq\left(\frac{L_{m}^{2}}{L_{s}}-L_{r}\right), \\
& \mathbf{A}_{r}(t) \triangleq\left[\begin{array}{cc}
R_{r} & \sigma \omega_{s} s(t) \\
-\sigma \omega_{s} s(t) & R_{r}
\end{array}\right], \\
& \mathbf{d}(t) \triangleq \frac{L_{m}}{L_{s}} s(t)\left[\begin{array}{c}
0 \\
V_{s}
\end{array}\right] .
\end{aligned}
$$

Proof. By substituting (10) and $R_{s}=0$ into (6), we have (11) as

$$
\mathbf{v}_{s}(t)=\left[\begin{array}{c}
0 \\
\omega_{s} \Psi_{s d}
\end{array}\right]=\left[\begin{array}{c}
0 \\
V_{s}
\end{array}\right],
$$

because $V_{s}=\left\|\mathbf{v}_{s}(t)\right\|=\left|\omega_{s} \Psi_{s d}\right|$. From (9) and (16), we have

$$
L_{s} \mathbf{i}_{s}(t)+L_{m} \mathbf{i}_{r}(t)=\frac{1}{\omega_{s}}\left[\begin{array}{c}
V_{s} \\
0
\end{array}\right] .
$$

Hence,

$$
\begin{aligned}
\mathbf{i}_{s}(t) & =-\frac{L_{m}}{L_{s}} \mathbf{i}_{r}(t)+\frac{1}{L_{s} \omega_{s}}\left[\begin{array}{c}
V_{s} \\
0
\end{array}\right], \\
\frac{\mathrm{d}}{\mathrm{d} t} \mathbf{i}_{s}(t) & =-\frac{L_{m}}{L_{s}} \frac{\mathrm{d}}{\mathrm{d} t} \mathbf{i}_{r}(t) .
\end{aligned}
$$

Substituting (18) and (19) into the second equation of (6), we have

$$
\begin{aligned}
\mathbf{v}_{r}(t) & =R_{r} \mathbf{i}_{r}(t)+\left(L_{r}-\frac{L_{m}^{2}}{L_{s}}\right) \frac{\mathrm{d}}{\mathrm{d} t} \mathbf{i}_{r}(t)+\omega_{s} s(t) \Theta \frac{L_{m}}{L_{s} \omega_{s}}\left[\begin{array}{c}
V_{s} \\
0
\end{array}\right] \\
& +\omega_{s} s(t) \Theta\left(L_{r}-\frac{L_{m}^{2}}{L_{s}}\right) \mathbf{i}_{r}(t) .
\end{aligned}
$$

From (7) and 20), we obtain (12).

The active power in the stator side of the DFIG $P_{s}$ can be calculated [25]

$$
\begin{aligned}
P_{s}(t) & =\operatorname{Re}\left\{\left(v_{s d}(t)+j v_{s q}(t)\right)\left(i_{s d}(t)-j i_{s q}(t)\right)\right\} \\
& =v_{s d}(t) i_{s d}(t)+v_{s q}(t) i_{s q}(t) .
\end{aligned}
$$

By substituting $v_{s d}(t)=0, v_{s q}(t)=V_{s}$ from 11 and $i_{s q}(t)=-\frac{L_{m}}{L_{s}} i_{r q}(t)$ from 18 into 21, we have

$$
P_{s}(t)=-\frac{L_{m}}{L_{s}} V_{s} i_{r q}(t) .
$$


When the power loss in the DFIG can be neglected, the power output of the generator $P_{e}$ is described by

$$
P_{e}(t)=P_{s}(t)+P_{r}(t)=(1-s(t)) P_{s}(t)
$$

where $P_{r}$ is the rotor-side active power. Hence,

$$
T_{e}(t)=\frac{P_{e}(t)}{\omega_{r}(t)}=\frac{p_{n} N P_{s}(t)}{\omega_{s}}=-\frac{p_{n} N L_{m}}{L_{s} \omega_{s}} V_{s} i_{r q}(t)
$$

\section{Controller design for rotor-side converter}

Assumption 2. We can measure $\mathbf{i}_{r}, \mathbf{i}_{s}, \mathbf{v}_{s}$, and $\omega_{r}$. We can also manipulate $v_{r d}, v_{r q}$, and the know the parameters $R_{s}$, $R_{r}, L_{s}, L_{r}, L_{m}, p_{n}$, and $N[1]$.

Assumption 3. The $d q / a b c$ transformation block, pulse-width modulation (PWM), and IGBT valves in the converters appropriately operate [1].

The objective of RSC is to maintain the $i_{r d}$ of the DFIG and the rotor speed $\omega_{r}$ of the wind turbine at the desired references. From (17, 12], and [24), to control $i_{r d}$ and $\omega_{r}$, we can adjust $i_{r d}$ and $i_{r q}$ by $v_{r d}$ and $v_{r q}$, respectively. To achieve this task, in previous research, traditional PI control was used [12, 26- 29]. In this research, a new law for rotor speed control is proposed.

Lemma 2. Under Assumptions 2 and 3 , for any reference $i_{r d r e f}$ and $\omega_{r \text { ref }}$, if $\mathbf{v}_{r}$ of the DFIG 6 is chosen as

$$
\begin{aligned}
\mathbf{v}_{r}(t)= & \mathbf{A}_{r}(t) \mathbf{i}_{r}(t)+\mathbf{d}(t)-\sigma \frac{\mathrm{d}}{\mathrm{d} t} \mathbf{i}_{\text {ref }}(t) \\
& -\sigma \mathbf{K}\left(\mathbf{i}_{r \text { ref }}(t)-\mathbf{i}_{r}(t)\right),
\end{aligned}
$$

where, for $k_{d}>0$,

$$
\begin{aligned}
& \mathbf{i}_{\text {ref }}(t) \triangleq\left[\begin{array}{c}
i_{r d \text { ref }}(t) \\
i_{r q \text { ref }}(t)
\end{array}\right]=\left[\begin{array}{c}
i_{r d \text { ref }}(t) \\
i_{r q}(t)+k_{d} \frac{\mathrm{d}}{\mathrm{d} t} e_{\omega_{\text {ref }}}(t)+k_{p} e_{\omega_{\text {ref }}}(t)
\end{array}\right], \\
& e_{\omega_{\text {ref }}}(t) \triangleq \omega_{\text {ref }}(t)-\omega_{r}(t),
\end{aligned}
$$

and if the feedback gain $\mathbf{K}$ and $k_{p}$ satisfy

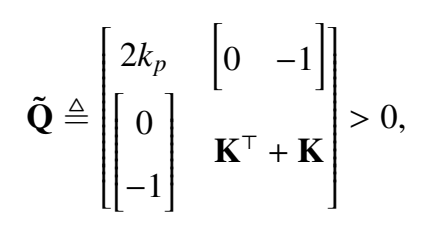

then

$$
\lim _{t \rightarrow \infty}\left(\mathbf{i}_{r \text { ref }}(t)-\mathbf{i}_{r}(t)\right)=0 \text {, and } \lim _{t \rightarrow \infty}\left(\omega_{r \text { ref }}(t)-\omega_{r}(t)\right)=0
$$


Remark 1. The inequality 28 means that $\tilde{\mathbf{Q}}=\tilde{\mathbf{Q}}^{\top}$ is a positive definite matrix. See Appendix A for the definition and the related properties.

Proof. Let us define

$$
\mathbf{e}_{1}(t) \triangleq\left[\begin{array}{c}
e_{\omega_{\text {ref }}}(t) \\
\mathbf{e}_{i}(t)
\end{array}\right]=\left[\begin{array}{c}
\omega_{\text {rref }}(t)-\omega_{r}(t) \\
\mathbf{i}_{\text {rref }}(t)-\mathbf{i}_{r}(t)
\end{array}\right]
$$

26) can be rewritten as

$$
k_{d} \frac{\mathrm{d}}{\mathrm{d} t} e_{\omega_{\text {ref }}}(t)=-k_{p} e_{\omega_{\text {ref }}}(t)+\left[\begin{array}{ll}
0 & 1
\end{array}\right] \mathbf{e}_{i}(t)
$$

By substituting (25) into (12), we have

$$
\frac{\mathrm{d}}{\mathrm{d} t}\left(\mathbf{i}_{r \mathrm{ref}}(t)-\mathbf{i}_{r}(t)\right)=-\mathbf{K}\left(\mathbf{i}_{r \mathrm{ref}}(t)-\mathbf{i}_{r}(t)\right) .
$$

Then,

$$
\mathbf{E}_{1} \frac{\mathrm{d}}{\mathrm{d} t} \mathbf{e}_{1}(t)=\mathbf{A}_{1} \mathbf{e}_{1}(t)
$$

where

$$
\mathbf{E}_{1}=\left[\begin{array}{cc}
k_{d} & \mathbf{0} \\
\mathbf{0} & \mathbf{I}_{2}
\end{array}\right]>0, \quad \mathbf{A}_{1}=-\left[\begin{array}{cc}
k_{p} & {\left[\begin{array}{cc}
0 & -1
\end{array}\right]} \\
\mathbf{0} & \mathbf{K}
\end{array}\right] .
$$

When we define a Lyapunov function as

$$
V_{1} \triangleq \mathbf{e}_{1}^{\top}(t) \mathbf{E}_{1} \mathbf{e}_{1}(t)
$$

its derivative is

$$
\dot{V}_{1}=\mathbf{e}_{1}^{\top}(t) \mathbf{E}_{1} \frac{\mathrm{d}}{\mathrm{d} t} \mathbf{e}_{1}(t)+\left(\frac{\mathrm{d}}{\mathrm{d} t} \mathbf{e}_{1}(t)\right)^{\top} \mathbf{E}_{1} \mathbf{e}_{1}(t) .
$$

By substituting 33 , into 36 , and noting that $\mathbf{A}_{1}+\mathbf{A}_{1}^{\top}=-\tilde{\mathbf{Q}}$, we have

$$
\begin{aligned}
\dot{V}_{1} & =\mathbf{e}_{1}^{\top}(t)\left(\mathbf{A}_{1}+\mathbf{A}_{1}^{\top}\right) \mathbf{e}_{1}(t)=-\mathbf{e}_{1}^{\top}(t) \tilde{\mathbf{Q}} \mathbf{e}_{1}(t) \\
& \leq-\lambda_{\min }(\tilde{\mathbf{Q}}) \mathbf{e}_{1}^{\top}(t) \mathbf{e}_{1}(t) .
\end{aligned}
$$

From the Lyapunov Stability Theory, $\lim _{t \rightarrow \infty} \mathbf{e}_{1}(t)=0$. This completes the proof.

Hence, from Lemma 2 and Assumption 3 if the rotor-side voltage of the DFIG is adjusted to satisfy [25], $\mathbf{i}_{r}(t)$ and $\omega_{r}(t)$ will converge to the desired values $\mathbf{i}_{\text {rref }}(t)$ and $\omega_{\text {rref }}(t)$, respectively.

Herein, we do not design a new control for the GSC. Instead, we use the control described in [1]. 


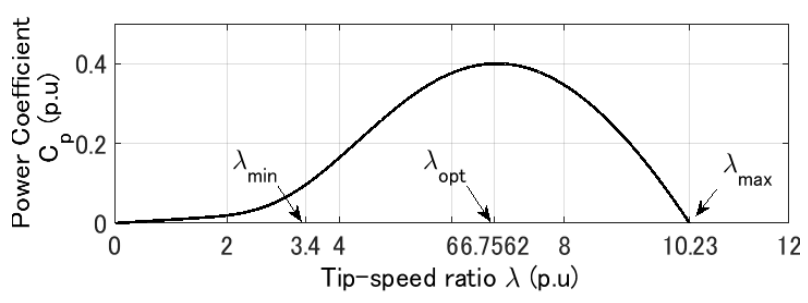

(a)

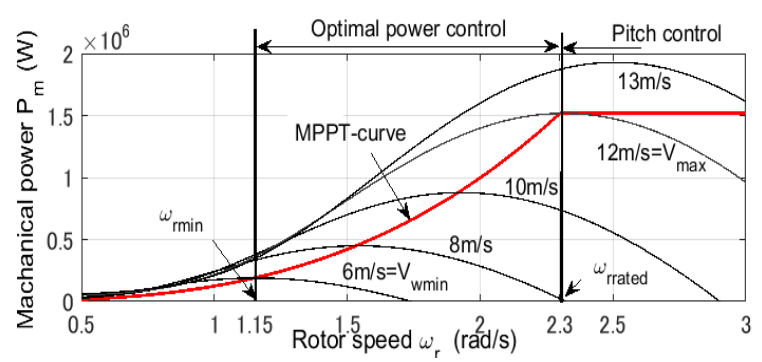

(b)

Fig. 2: Wind turbine characteristic of 41 for $\beta=0$ : (a) $C_{p}(\lambda)$, (b) $P_{m}\left(\lambda, V_{w}\right)$, and $P_{\mathrm{mppt}}\left(\omega_{r}\right)$.

\section{Maximum power point tracking scheme}

The wind turbine is operated in the optimal power control region defined with the minimum rotor speed $\omega_{r \min }$, rated rotor speed $\omega_{\text {rrated }}$, minimum wind speed $V_{w \min }$, and rated wind speed $V_{w r a t e d}$ by

$$
\begin{aligned}
D \triangleq\left\{\left(\omega_{r}, V_{w}\right) \mid \omega_{r \min }\right. & \leq \omega_{r} \leq \omega_{\text {rated }}, \\
V_{w \min } & \left.\leq V_{w} \leq V_{w \text { rated }}, \text { and } C_{p}(\lambda)>0\right\}
\end{aligned}
$$

In the optimal power control region $D$, the tip-speed ratio is bounded as

$$
\lambda_{\min } \triangleq \frac{R \omega_{\text {rmin }}}{V_{w \text { rated }}} \leq \lambda(t) \leq \lambda_{\max } \triangleq \max \left\{\lambda \mid C_{p}(\lambda)>0\right\} .
$$

This paper aims to suggest a MPPT scheme and controller such that the wind turbine can work in the optimal power control region $D$ of the MPPT curve.

\subsection{MPPT-curve method}

We consider a maximization of the mechanical power to change $\omega_{r}$. Evidently, it is equivalent to a maximization of $C_{p}\left(\lambda\left(\omega_{r}, V_{w}\right)\right)$. That is,

$$
\begin{aligned}
C_{p \max } & \triangleq C_{p}\left(\lambda_{\mathrm{opt}}\right), \\
\lambda_{\mathrm{opt}} & \triangleq \arg \max _{\lambda} C_{p}(\lambda) .
\end{aligned}
$$

In this paper, we use

$$
C_{p}(\lambda)=\left(165.2842 \lambda^{-1}-16.8693\right) e^{-21 \lambda^{-1}}+0.009 \lambda
$$

for the pitch angle $\beta=0$. It has a unique maximum point of $C_{p \max }=0.4$ at $\lambda_{\mathrm{opt}}=6.7562$ (Fig. 2 $)$ ).

The optimal rotor speed

$$
\omega_{\mathrm{opt}}\left(V_{w}\right) \triangleq \frac{\lambda_{\mathrm{opt}} V_{w}}{R} .
$$


achieves the maximal mechanical power

$$
\begin{aligned}
\max _{\omega_{r}} P_{m}\left(\omega_{r}, V_{w}\right) & =\frac{1}{2} \rho \pi R^{2} C_{p \max } V_{w}^{3} \\
& =k_{\mathrm{opt}} \omega_{\mathrm{opt}}^{3}\left(V_{w}\right), \\
k_{\mathrm{opt}} & \triangleq \frac{1}{2} \rho \pi R^{5} \frac{C_{p \max }}{\lambda_{\mathrm{opt}}^{3}} .
\end{aligned}
$$

To maximize the mechanical power, if we have a wind speed $V_{w}$, we simply control to make $\omega_{r}(t)$ track the $\omega_{\text {opt }}\left(V_{w}(t)\right)$ given in 42. However, since it is difficult to obtain precise values of $V_{w}$, we generally control $\omega_{r}$ to make the mechanical power $P_{m}\left(\omega_{r}, V_{w}\right)$ track

$$
P_{\mathrm{mppt}}\left(\omega_{r}\right)=k_{\mathrm{opt}} \omega_{r}^{3}
$$

instead of 44. $P_{\text {mppt }}\left(\omega_{r}\right)$ is a locus of the peak of $P_{m}\left(\omega_{r}, V_{m}\right)$ as $V_{m}$ changes in the optimal power control region $D$ (Fig. 2p). This is called the MPPT-curve method or MPPT scheme.

\subsection{Proposed MPPT control}

In this subsection, we propose a new MPPT scheme using no real-time information about $V_{w}(t)$. The scheme aims to reduce $\left|\omega_{\text {ropt }}\left(V_{w}(t)\right)-\omega_{r}(t)\right|$ to achieve the maximum $P\left(\omega_{r}, V_{w}\right)$.

Although the scheme does not use any real-time measurement of $V_{w}$, as in [1], for analysis of the control performance, we need the bound of the wind speed as

$$
\frac{\mathrm{d}}{\mathrm{d} t} \omega_{r \mathrm{opt}}\left(V_{w}(t)\right)=\frac{\lambda_{\mathrm{opt}}}{R} \frac{\mathrm{d}}{\mathrm{d} t} V_{w}(t) \leq \frac{\lambda_{\mathrm{opt}}}{R}\left|\frac{\mathrm{d}}{\mathrm{d} t} V_{w}(t)\right| \triangleq \gamma .
$$

Moreover, in the analysis of the proposed scheme, we use

$$
\zeta\left(\omega_{r}, V_{w}\right) \triangleq-\frac{P_{m}\left(\omega_{r}, V_{w}\right)-P_{\mathrm{mppt}}\left(\omega_{r}\right)}{\omega_{r}\left(\omega_{r}-\omega_{\text {ropt }}\left(V_{w}\right)\right)} .
$$

This is well defined by L'Hôpital's rule to ensure that

$$
\begin{aligned}
& \lim _{\omega_{r} \rightarrow \omega_{\text {ropt }}\left(V_{w}\right)} \zeta\left(\omega_{r}, V_{w}\right) \\
& =-\lim _{\omega_{r} \rightarrow \omega_{\text {ropt }}\left(V_{w}\right)} \frac{\frac{\mathrm{d}}{\mathrm{d} \omega_{r}} P_{m}\left(\omega_{r}, V_{w}\right)-\frac{\mathrm{d}}{\mathrm{d} \omega_{r}} P_{\mathrm{mppt}}\left(\omega_{r}\right)}{\frac{\mathrm{d}}{\mathrm{d} \omega_{r}}\left(\omega_{r}\left(\omega_{r}-\omega_{r \mathrm{opt}}\left(V_{w}\right)\right)\right)} \\
& =-\lim _{\omega_{r} \rightarrow \omega_{\text {ropt }}\left(V_{w}\right)} \frac{\frac{R}{V_{w}} \frac{\partial}{\partial \lambda} P_{m}\left(\lambda, V_{w}\right)-3 k_{\mathrm{opt}} \omega_{r}^{2}}{2 \omega_{r}-\omega_{\text {ropt }}\left(V_{w}\right)} \\
& =\frac{3 k_{\mathrm{opt}} \omega_{r \mathrm{opt}}\left(V_{w}\right)^{2}}{\omega_{r \mathrm{opt}}\left(V_{w}\right)}=3 k_{\mathrm{opt}} \omega_{r \mathrm{opt}}\left(V_{w}\right) .
\end{aligned}
$$


Assumption 4. The precise value of $k_{\mathrm{opt}}$ for the MPPT curve is not available. Instead, we can use the estimate $k_{\mathrm{opt}}^{\prime}$ with

$$
k_{\mathrm{opt}}^{\prime}=(1+\delta) k_{\mathrm{opt}}, \quad|\delta| \leq \delta_{\max }
$$

The proposed MPPT scheme is given as the reference [27) for the RSC control 25] as

$$
\begin{aligned}
\omega_{\mathrm{rref}}(t) & \triangleq\left(\frac{\hat{P}_{\mathrm{mppt}}(t)}{\hat{k}_{\mathrm{opt}}(t)}\right)^{1 / 3}, \\
\hat{P}_{\mathrm{mppt}}(t) & =\omega_{r}(t)\left(k_{1} \frac{\mathrm{d}}{\mathrm{d} t} \omega_{r}(t)-k_{2}\left(\omega_{r}(t)-\hat{\omega}_{r \mathrm{opt}}(t)\right)\right)+P_{e}(t), \\
\frac{\mathrm{d}}{\mathrm{d} t} \hat{\omega}_{\mathrm{ropt}}(t) & \triangleq k_{3}\left(\omega_{r}(t)-\hat{\omega}_{\mathrm{ropt}}(t)\right) \\
\frac{\mathrm{d}}{\mathrm{d} t} \hat{k}_{\mathrm{opt}}(t) & \triangleq k_{4}\left(k_{\mathrm{opt}}^{\prime}-\hat{k}_{\mathrm{opt}}(t)\right)+\omega_{r}(t)^{2}\left(\omega_{r}(t)-\hat{\omega}_{r \mathrm{opt}}(t)\right),
\end{aligned}
$$

where $\hat{k}_{\mathrm{opt}}(t)$ and $\hat{\omega}_{\text {ropt }}(t)$ are estimations of $k_{\mathrm{opt}}$ and $\omega_{\text {ropt }}\left(V_{w}(t)\right)$, respectively. The feedback gains $k_{1}, k_{2}, k_{3}$, and $k_{4}$ are designed as the conditions in Theorem 1 and

$$
J>k_{1} \geq 0
$$

Lemma 3. In the optimal power control region $D, \hat{k}_{\mathrm{opt}}(t)$ is bounded, i.e.,

$$
\begin{aligned}
& \max \hat{k}_{\mathrm{opt}}(t) \leq \hat{k}_{\mathrm{opt}, \mathrm{ub}} \\
& \hat{k}_{\mathrm{opt}, \mathrm{ub}}=2 k_{4}^{-1} \omega_{\text {rrated }}^{3}+k_{4}^{-1}\left|\hat{\omega}_{\text {ropt }}(0)\right| \omega_{\text {rrated }}^{2}+\left|\hat{k}_{\mathrm{opt}}(0)\right|+k_{\mathrm{opt}}^{\prime}
\end{aligned}
$$

See Appendix B for the proof.

Theorem 1. In addition to Assumption 4, we suppose that 51) is restricted within the optimal control region $D$ as

$$
\left(\omega_{r \mathrm{ref}}(t), V_{w}\right) \in D
$$

Then, if there exist positive constants $\alpha, v, w$ and $q$ satisfying

$$
\left\{\begin{array}{l}
\boldsymbol{\Xi}=\mathbf{K}^{\top}+\mathbf{K}-q \mathbf{I}_{2}>0 \\
2 k_{p}-\alpha \hat{k}_{\mathrm{opt}, \mathrm{ub}}^{2} \xi_{\mathrm{max}}^{2}-\left[\begin{array}{ll}
0 & 1
\end{array}\right] \boldsymbol{\Xi}^{-1}\left[\begin{array}{l}
0 \\
1
\end{array}\right]-q k_{d}>0, \\
2 \zeta_{\mathrm{min}}-(w \gamma+q) \hat{J}-\left(k_{3}-k_{2}\right)-1>0 \\
k_{3}-k_{2}-\omega_{\text {rrated }}^{2}-w \gamma-q>0 \\
\left(2-v k_{\mathrm{opt}}\right) k_{4}-\omega_{\text {rrated }}^{2}-q>0
\end{array}\right.
$$


where

$$
\begin{aligned}
\zeta_{\min } & \triangleq \min \zeta\left(\omega_{r}, V_{w}\right), \\
\xi\left(\omega_{r}, \omega_{r \mathrm{ref}}\right) & \triangleq \omega_{r}^{-1} \omega_{r \text { ref }}^{2}+\omega_{r}+\omega_{r \mathrm{ref}}, \\
\xi_{\max } & \triangleq \max \xi\left(\omega_{r}, \omega_{r \mathrm{ref}}\right), \\
\hat{J} & \triangleq J-k_{1}>0,
\end{aligned}
$$

then, there exists a time $t_{o}>0$ such that for all $t \geq t_{o}$,

$$
\left|\omega_{r}(t)-\omega_{\text {ropt }}\left(V_{w}(t)\right)\right|<\frac{1}{\sqrt{q}} \sqrt{\frac{1+\hat{J}^{-1}}{w} \gamma+\frac{k_{4} \hat{J}^{-1}}{v} k_{\mathrm{opt}} \delta_{\max }^{2}} .
$$

See Appendix C for the proof.

Remark 2. We consider $\xi\left(\omega_{r}, \omega_{r \text { ref }}\right)$ only for the region of $\omega_{r}>0$ and $\omega_{r \text { ref }}>0$, and the Hessian of $\xi\left(\omega_{r}, \omega_{r \mathrm{ref}}\right)$ is positive semidefinite as

$$
\left[\begin{array}{cc}
\frac{\partial^{2} \xi}{\partial \omega_{r}^{2}} & \frac{\partial^{2} \xi}{\partial \omega_{r} \partial \omega_{r \text { ref }}} \\
\frac{\partial^{2} \xi}{\partial \omega_{r \mathrm{ref}} \partial \omega_{r}} & \frac{\partial^{2} \xi}{\partial \omega_{r \text { ref }}^{2}}
\end{array}\right]=2\left[\begin{array}{cc}
\frac{\omega_{r \mathrm{ref}}^{2}}{\omega_{r}^{3}} & -\frac{\omega_{\text {rref }}}{\omega_{r}^{2}} \\
-\frac{\omega_{r \mathrm{rref}}}{\omega_{r}^{2}} & \frac{1}{\omega_{r}}
\end{array}\right] \geq 0 .
$$

Hence, $\xi_{\max }$ is attained at the boundary of the region $\left[\omega_{r \min }, \omega_{r \text { rated }}\right] \times\left[\omega_{r \min }, \omega_{r \text { rated }}\right]$. Moreover, from

$$
\left[\begin{array}{c}
\frac{\partial \xi}{\partial \omega_{r}} \\
\frac{\partial \xi}{\partial \omega_{r \mathrm{ref}}}
\end{array}\right]=\left[\begin{array}{c}
1-\frac{\omega_{r \mathrm{ref}}^{2}}{\omega_{r}^{2}} \\
1+2 \frac{\omega_{r \mathrm{ref}}}{\omega_{r}}
\end{array}\right]
$$

it is easy to realize $\xi_{\max }=\xi\left(\omega_{r \min }, \omega_{r \text { rated }}\right)$.

Note that the optimal control region $D$ is divided into three parts:

$$
\begin{aligned}
D_{\mathrm{lr}} & \triangleq\left\{\left(\omega_{r}, V_{w}\right) \in D \mid R \omega_{r} / V_{w}>\lambda_{\mathrm{opt}}\right\} \\
D_{\mathrm{opt}} & \triangleq\left\{\left(\omega_{r}, V_{w}\right) \in D \mid R \omega_{r} / V_{w}=\lambda_{\mathrm{opt}}\right\} \\
D_{\mathrm{ul}} & \triangleq\left\{\left(\omega_{r}, V_{w}\right) \in D \mid R \omega_{r} / V_{w}<\lambda_{\mathrm{opt}}\right\} .
\end{aligned}
$$

Note also that $\left(\omega_{r}, V_{w \mathrm{mppt}}\left(\omega_{r}\right)\right) \in D_{\text {opt }}$ and $\left(\omega_{\text {ropt }}\left(V_{w}\right), V_{w}\right) \in D_{\text {opt }}$. Moreover,

$$
\frac{\partial}{\partial \lambda} C_{p}(\lambda) \begin{cases}<0 & \left(\omega_{r}, V_{w}\right) \in D_{\mathrm{lr}} \\ =0 & \left(\omega_{r}, V_{w}\right) \in D_{\mathrm{opt}} \\ >0 & \left(\omega_{r}, V_{w}\right) \in D_{\mathrm{ul}} .\end{cases}
$$

By regarding (5) to use (4) as

$$
P_{m}\left(\omega_{r}, \lambda\right)=\frac{1}{2} \rho \pi R^{5} \frac{C_{p}(\lambda)}{\lambda^{3}} \omega_{r}^{3}
$$




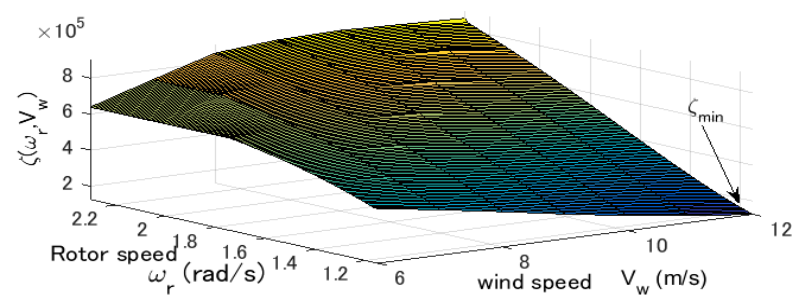

Fig. 3: $\zeta\left(\omega_{r}, V_{w}\right)$.

and as in [1], from $P_{\mathrm{mppt}}\left(\omega_{r}\right)=k_{\mathrm{opt}} \omega_{r}^{3}$ and the definition of $k_{\mathrm{opt}}$ in 45 , we have

$$
P_{m}\left(\omega_{r}, V_{w}\right)-P_{\mathrm{mptt}}\left(\omega_{r}\right)=\frac{1}{2} \rho \pi R^{5} \omega_{r}^{3}\left(\frac{C_{p}(\lambda)}{\lambda^{3}}-\frac{C_{p \max }}{\lambda_{\mathrm{opt}}^{3}}\right) .
$$

Hence,

$$
\begin{aligned}
\zeta\left(\omega_{r}, V_{w}\right) & =\frac{\rho \pi R^{5} \omega_{r}^{2}}{2\left(\omega_{r}-\omega_{\text {ropt }}\left(V_{w}\right)\right)}\left(\frac{C_{p \max }}{\lambda_{\mathrm{opt}}^{3}}-\frac{C_{p}(\lambda)}{\lambda^{3}}\right) \\
& =\frac{\rho \pi R^{6} \omega_{r}^{2}}{2 V_{w} \lambda^{3}\left(\lambda-\lambda_{\mathrm{opt}}\right)}\left(\frac{C_{p \max }}{\lambda_{\mathrm{opt}}^{3}} \lambda^{3}-C_{p}(\lambda)\right) .
\end{aligned}
$$

Lemma 4. For a point $\left(\omega_{r}, V_{w}\right) \in D_{\mathrm{opt}} \cup D_{\mathrm{lr}}, \zeta\left(\omega_{r}, V_{w}\right)>0$. Moreover, for a point $\left(\omega_{r}, V_{w}\right) \in D_{\mathrm{ul}}$, if

$$
\frac{C_{p \max }}{\lambda_{\mathrm{opt}}^{3}}-\frac{C_{p}(\lambda)}{\lambda^{3}}<0
$$

then

$$
\zeta\left(\omega_{r}, V_{w}\right)>0
$$

Proof. From (49), for a point $\left(\omega_{r}, V_{w}\right) \in D_{\mathrm{opt}}, \zeta\left(\omega_{r}, V_{w}\right)>0$.

For a point $\left(\omega_{r}, V_{w}\right) \in D_{\mathrm{lr}}$, since $\lambda>\lambda_{\mathrm{opt}}$, from (74), $C_{p}(\lambda) \leq C_{p \max }$,

$$
\zeta\left(\omega_{r}, V_{w}\right)>\frac{\rho \pi R^{6} \omega_{r}^{2} C_{p}(\lambda)}{2 V_{w} \lambda^{3}\left(\lambda-\lambda_{\mathrm{opt}}\right)}\left(\frac{\lambda^{3}}{\lambda_{\mathrm{opt}}^{3}}-1\right)>0 .
$$

For a point $\left(\omega_{r}, V_{w}\right) \in D_{\mathrm{ul}}$, since $\omega_{r}<\omega_{\text {ropt }}\left(V_{w}\right)$, it follows from $(73)$ and $(75)$ that $\zeta\left(\omega_{r}, V_{w}\right)>0$.

\section{Simulation results}

In this section, we evaluate the performance of the proposed MPPT scheme by comparing the simulation results for the 1.5 MW DFIG wind turbine with the conventional MPPT-curve method with traditional PI control. In all simulations, we used the parameters of the wind turbine [1] and generator [24] as shown in Table 1] 
Table 1: Parameters in simulations (from [1] for wind turbine parameters and [24] for DFIG parameters)

\begin{tabular}{|c|c|c|c|}
\hline Name & Symbol & Value & Unit \\
\hline Rated power & $P$ & 1.5 & MW \\
\hline The length of blade & $R$ & 35.25 & $\mathrm{~m}$ \\
\hline Normal rotor speed & $\omega_{\text {rrated }}$ & 22 & rpm \\
\hline Minimum rotor speed & $\omega_{r \min }$ & 11 & $\mathrm{rpm}$ \\
\hline Rated wind speed & $V_{w \text { rated }}$ & 12 & $\mathrm{~m} / \mathrm{s}$ \\
\hline Rated stator voltage & $V_{s}$ & 690 & $\mathrm{~V}$ \\
\hline Rated rotor voltage & $V_{r}$ & 120 & $\mathrm{~V}$ \\
\hline Rated stator frequency & $f$ & 50 & $\mathrm{~Hz}$ \\
\hline Minimum rotor speed & $\Omega_{r \min }$ & 1200 & $\mathrm{rpm}$ \\
\hline Rated rotor speed & $\Omega_{r \text { rated }}$ & 1750 & $\mathrm{rpm}$ \\
\hline Number of pole pairs & $p_{n}$ & 2 & p.u \\
\hline Stator winding resistance & $R_{s}$ & 2.65 & $\mathrm{~m} \Omega$ \\
\hline Rotor winding resistance & $R_{r}$ & 2.63 & $\mathrm{~m} \Omega$ \\
\hline Stator winding inductance & $L_{s}$ & 5.6438 & $\mathrm{mH}$ \\
\hline Rotor winding inductance & $L_{r}$ & 5.6068 & $\mathrm{mH}$ \\
\hline Magnetizing inductance & $L_{m}$ & 5.4749 & $\mathrm{mH}$ \\
\hline Gearbox ratio & $N$ & 79.545 & p.u \\
\hline Inertia of system & $J$ & 445000 & $\mathrm{~kg} \cdot \mathrm{m}^{2}$ \\
\hline
\end{tabular}


We used the power coefficient 411 by setting $\beta=0$. Then,

$$
\begin{aligned}
& k_{\mathrm{opt}}=1.2467 \times 10^{5} \mathrm{~kg} \cdot \mathrm{m}^{2} \\
& \lambda_{\mathrm{opt}}=6.7562 .
\end{aligned}
$$

As in [1], we used the optimal control region $D$ defined by

$$
\begin{aligned}
\omega_{r \min } & =1.15 \mathrm{rad} / \mathrm{s}, \quad \omega_{r \text { rated }}=2.3 \mathrm{rad} / \mathrm{s}, \\
V_{w \min } & =\frac{R \omega_{r \min }}{\lambda_{\text {opt }}}=\frac{35.25 \times 1.15}{6.7562}=6 \mathrm{~m} / \mathrm{s} \\
V_{\text {wrated }} & =12 \mathrm{~m} / \mathrm{s} .
\end{aligned}
$$

The wind speed profile Fig. 40 we used satisfied $V_{w}(t)<V_{w r a t e d}$, and $\left|\frac{\mathrm{d}}{\mathrm{d} t} V_{w}(t)\right| \leq 0.44 \mathrm{~m} / \mathrm{s}^{2}$. Hence,

$$
\gamma=\frac{\lambda_{\mathrm{opt}} 0.44}{R}=\frac{6.7562 \times 0.44}{35.25}=0.0843
$$

Hence, from (47) and 61], we have

$$
\begin{aligned}
\max \xi\left(\omega_{r}, \omega_{r \mathrm{ref}}\right) & =\frac{\omega_{r \mathrm{rated}}^{2}}{\omega_{r \min }}+\omega_{r \min }+\omega_{r \text { rated }} \\
& =\frac{2.3^{2}}{1.15}+1.15+2.3=8.05
\end{aligned}
$$

In $D, \lambda_{\max }=10.239$ and

$$
\lambda_{\min }=\frac{R \omega_{\text {rmin }}}{V_{\text {wrated }}}=\frac{35.25 \times 1.15}{12}=3.4 \text { p.u. }
$$

Obviously, since the power coefficient (41) satisfies the condition (75) in Lemma $4, \zeta\left(\omega_{r}, V_{w}\right)$ is always positive, as shown in Fig. 3 From this figure,

$$
\min _{\left(\omega_{r}, V_{w}\right) \in D} \zeta\left(\omega_{r}, V_{w}\right)=1.271 \times 10^{5} .
$$

The reference values setting for the RSC control 25$]$ with $i_{r d \text { ref }}=401.4 \mathrm{~A}$,

$$
\mathbf{K}=200 \mathbf{I}_{2} \text {. }
$$

Note that as $i_{r d}(t) \rightarrow 401.4$ A, the DFIG will generate with a unity power factor. Here, we used $\omega_{\text {rref }}(t)$ with

$$
\begin{aligned}
& k_{1}=0.3 J, \hat{J}=J-k_{1}=0.7 J=3.12 \times 10^{5}, \\
& k_{2}=2 \hat{J}, k_{3}=k_{2}+0.001 \hat{J}, k_{4}=10, k_{d}=0.0029 J, k_{p}=100 k_{d}, \\
& \hat{\omega}_{\text {ropt }}(0)=\omega_{\text {rrated }}, k_{\mathrm{opt}}^{\prime}=124610, \delta_{\mathrm{max}}=5 k_{\mathrm{opt}} \times 10^{-4}, \\
& \hat{k}_{\mathrm{opt}}(0)=k_{\mathrm{opt}}^{\prime}, \hat{k}_{\mathrm{opt}, \mathrm{ub}}=k_{\mathrm{opt}}^{\prime}+k_{\mathrm{opt}}^{\prime}+3 k_{4}^{-1} \omega_{\text {rrated }}^{3} \approx 2 k_{\mathrm{opt}} .
\end{aligned}
$$

Hence, when $v=0.6 \times 10^{-5}, w=4.825, \alpha=k_{d} \hat{k}_{\mathrm{opt}, \mathrm{ub}}^{-2} \xi_{\max }^{-2}$ and $q=0.4$ 


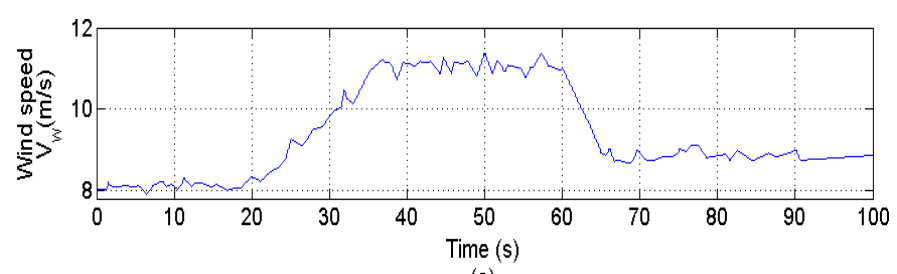

(a)

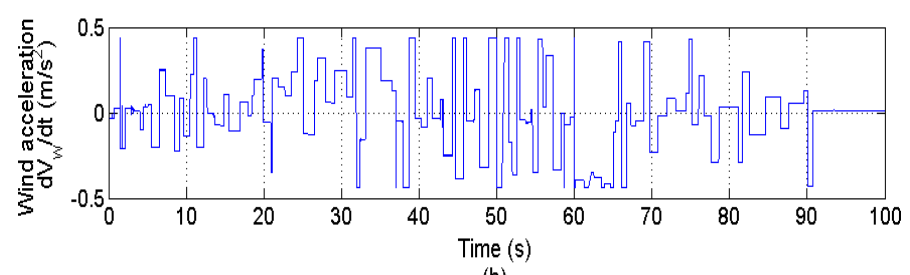

(b)

Fig. 4: Wind speed profile: (a) wind speed and (b) wind acceleration.

$$
\begin{aligned}
& \boldsymbol{\Xi}=\mathbf{K}^{\top}+\mathbf{K}-q \mathbf{I}_{2}=\operatorname{diag}(399.6,399.6)>0 \\
& {\left[\begin{array}{ll}
0 & 1
\end{array}\right] \boldsymbol{\Xi}^{-1}\left[\begin{array}{l}
0 \\
1
\end{array}\right]+q k_{d}=0.0025+0.4 k_{d} \approx 0.4 k_{d}} \\
& 2 k_{p}-\alpha \hat{k}_{\mathrm{opt}, \mathrm{ub}}^{2} \xi_{\max }^{2}-\left[\begin{array}{ll}
0 & 1
\end{array}\right] \boldsymbol{\Xi}^{-1}\left[\begin{array}{l}
0 \\
1
\end{array}\right]-q k_{d} \approx 198.6 k_{d}>0
\end{aligned}
$$

and

$$
\begin{gathered}
2 \zeta_{\min }-w \hat{J} \gamma-\left(k_{3}-k_{2}\right)-1 \approx 2.542 \times 10^{5}-0.105 w \hat{J}-0.001 \hat{J} \\
=(0.8145-0.0843 w-0.001) \hat{J} \\
=0.4068 \hat{J}>q \hat{J}, \\
k_{3}-k_{2}-\omega_{\text {rated }}^{2}-w \gamma=0.001 \hat{J}-2.3^{2}-0.0843 w \\
\approx 3.12 \times 10^{2}-5.6967=306.3033>q, \\
\left(2-v k_{\text {opt }}\right) k_{4}-\omega_{\text {rated }}^{2}=\left(2-v \times 1.2467 \times 10^{5}\right) k_{2}-2.3^{2} \\
=6.26-5.29=0.97>q .
\end{gathered}
$$

It is easily observed that the five inequalities in Theorem 1 are satisfied. Hence, the upper bound for the rotor speed $\omega_{r}(t)$ in Theorem 1 is

$$
\begin{aligned}
& \left|\omega_{r}(t)-\omega_{\text {ropt }}\left(V_{w}(t)\right)\right| \leq \\
& \frac{1}{\sqrt{0.4}} \sqrt{\frac{0.0843}{4.825}\left(1+\frac{1}{3.12 \times 10^{5}}\right)+\frac{5 \times 25 \times 10^{-8}}{0.6 \times 10^{-5}} \frac{1.2467 \times 10^{5}}{3.12 \times 10^{5}}} \\
& =0.254 .
\end{aligned}
$$




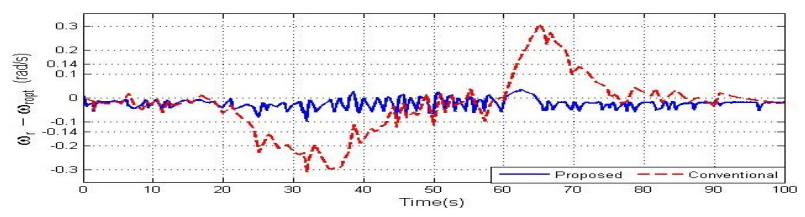

(a)

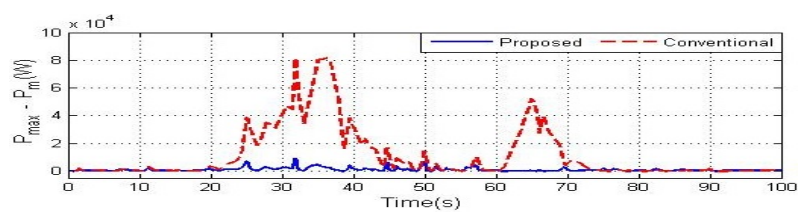

(c)

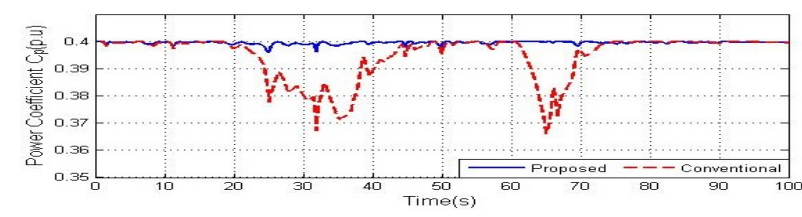

(b)

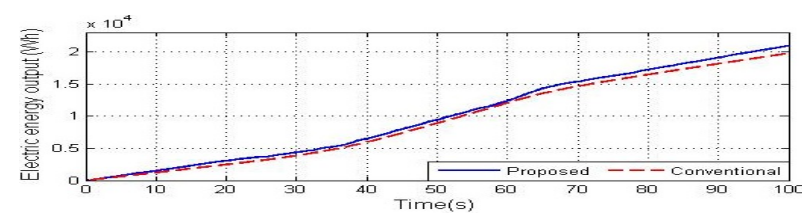

(d)

Fig. 5: Simulation results: (a) $\omega_{r}(t)-\omega_{\text {ropt }}\left(V_{w}(t)\right)$, (b) power coefficient $C_{p}(\lambda(t))$, (c) $P_{\max }(t)-P_{m}(t)$, and (d) electrical energy output.

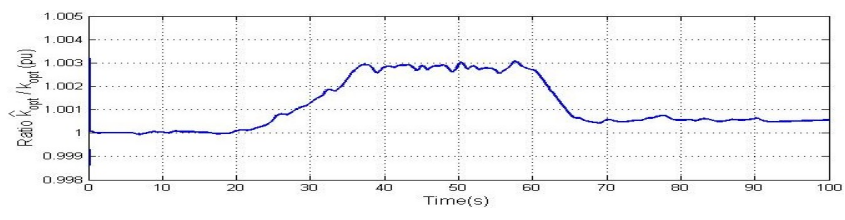

(a)

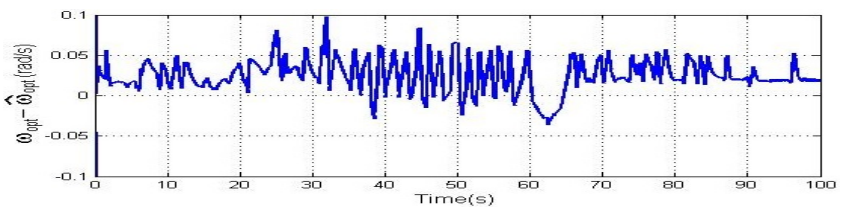

(b)

Fig. 6: Simulation results: (a) ratio $\hat{k}_{\mathrm{opt}} / k_{\mathrm{opt}}$ and (b) $\omega_{\text {ropt }}(t)-\hat{\omega}_{\text {ropt }}(t)$.

For the above DFIG wind turbine, wind profile, and controller, the simulation results are shown in Fig. 5 , Fig. 5 a argues that with the conventional method, the error between $\omega_{r}(t)$ and $\omega_{\text {ropt }}(t)$ is still quite large, up to $0.3 \mathrm{rad} / \mathrm{s}$. This is unlikely with the proposed method, as $\omega_{r}(t)$ always approaches $\omega_{\text {ropt }}(t)$ and guarantees that the $\left|\omega_{r}(t)-\omega_{\text {ropt }}(t)\right|$ is always very small, below $0.254 \mathrm{rad} / \mathrm{s}$, as Theorem 1 . Consequently, the power coefficient $C_{p}$ is virtually maintained around its maximum value $C_{p \max }=0.4$ p.u. during the simulation interval. This is displayed clearly by the solid line in Fig. 5b. This performance is hardly seen in the case with the conventional method, because during the interval of rapid decrease in wind speed, the large error in rotor speed, $\omega_{r}(t)-\omega_{\text {ropt }}(t)$, leads to a reduction of $C_{p}$ to 0.45 p.u., as shown by the broken line in Fig. 5 p.

Concerning the mechanical power output of the wind turbine, Fig. 5f depicts the error between $P_{\max }$ and $P_{m}$. The figure shows that when the wind velocity varies dramatically, the error between $P_{\max }$ and $P_{m}$ is approximately to zero with the proposed method. This is mainly because the power coefficient $C_{p}$ remains around $C_{p \max }$, as shown in Fig. 5b. In other words, with the proposed method, the main objective, which is to have $P_{m}$ approach $P_{\max }$, is completely achieved. With the conventional method, however, this goal is not achievable due to the significant decrease in $C_{p}$ during sudden variations in wind conditions.

With the proposed strategy, the total electrical energy output of the generator is higher than that with the conven- 


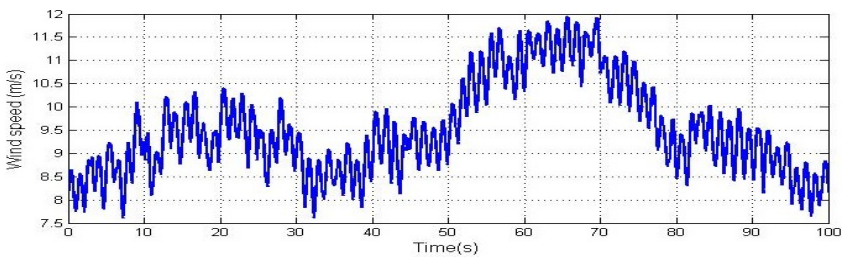

(a)

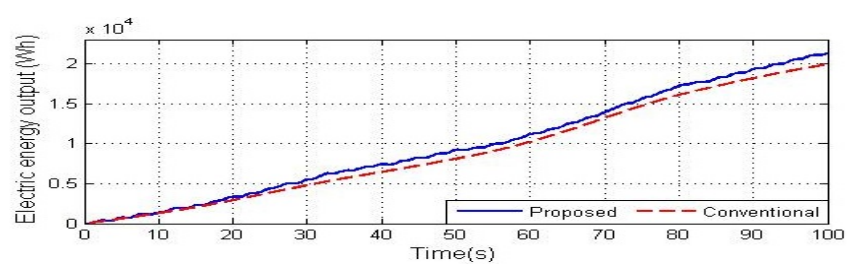

(c)

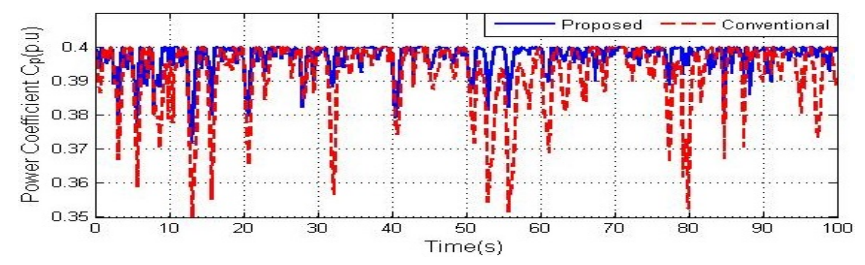

(b)

Fig. 7: Simulation results: (a) wind speed, (b) power coefficient, and (c) energy output.

tional strategy, as shown in Fig. 5d. This is mainly because $P_{m}$ in the case of the proposed method has a higher value. This confirms that the quality of the proposed scheme is always better than that of the conventional one.

Fig. 6 indicates that during the simulation interval, $\hat{k}_{\text {opt }}$ is always below its maximum value, which is estimated as $\hat{k}_{\text {opt,ub }}=2 k_{\text {opt }}$. From Fig. 6 , the gap between $\omega_{\text {ropt }}(t)$ and its estimation $\hat{\omega}_{\text {ropt }}(t)$ is quite small, below $0.097 \mathrm{rad} / \mathrm{s}$. In other words, the proposed method is more suitable than the conventional one.

In the case where a rapid wind profile, similar to that shown in Fig. 7 a, is used, the simulation results are demonstrated in Fig. 7p and Fig. 7f. Obviously, the wind turbine with the proposed method has better performance than with the conventional one, in terms of both the power coefficient $C_{p}$ and energy output.

\section{Conclusion}

This paper suggests a MPPT method for DFIG wind turbines without any information about the wind data or an available anemometer. With the proposed MPPT method, it is guaranteed that a wind turbine can track the maximum power operation point better than a wind turbine with the conventional MPPT-curve method; this is verified through the simulation of a 1.5-MW DFIG wind turbine in a MATLAB/Simulink environment. The simulation results illustrate that $C_{p}, P_{m}$, and $\omega_{r}$ vary around their optimal values; the electrical energy output of the generator is always higher than that extracted with the conventional method. Furthermore, with the proposed control method, the wind turbine is always in stable operation. Thus, the proposed control method has a high qualified performance.

\section{Appendix A. Matrix inequality}

Let $\mathbf{S}^{n}$ denote a set of real-valued symmetrical $n \times n$ matrices and let $\lambda_{i}(\mathbf{Y})$ denote the $i$ th eigenvalue of $\mathbf{Y} \in \mathbf{S}^{n}$. 
For $\mathbf{Y} \in \mathbf{S}^{n}$ and $\mathbf{x} \in \mathbf{R}^{n}$, the following inequality holds [30]:

$$
\min \lambda_{i}(\mathbf{Y})\|\mathbf{x}\|^{2} \leq \mathbf{x}^{\top} \mathbf{Y} \mathbf{x} \leq \max \lambda_{i}(\mathbf{Y})\|\mathbf{x}\|^{2} .
$$

Definition 1. (ex. p. 647 in [31]) A matrix $\mathbf{Y}=\mathbf{Y}^{\top} \in \mathbf{R}^{n \times n}$ is said to be positive definite if $\mathbf{x}^{\top} \mathbf{Y} \mathbf{x}>0$ for all nonzero vector $\mathbf{x} \in \mathbf{R}^{n}$. We denote the positive definite matrix as $\mathbf{Y}>0$. Moreover, if $\mathbf{x}^{\top} \mathbf{Y} \mathbf{x} \geq 0$ for all $\mathbf{x} \neq 0, \mathbf{Y}$ is said to be positive semidefinite, and we denote it as $\mathbf{Y} \geq 0$.

Lemma 5. For $\mathbf{Y} \in \mathbf{S}^{n}$,

$$
\begin{gathered}
\mathbf{Y}>0 \quad \Leftrightarrow \quad \lambda_{i}(\mathbf{Y})>0, \\
\mathbf{Y} \geq 0 \quad \Leftrightarrow \quad \lambda_{i}(\mathbf{Y}) \geq 0 .
\end{gathered}
$$

For $\mathbf{Y}, \mathbf{Z} \in \mathbf{S}^{n}$, we use $\mathbf{Y}>(\geq) \mathbf{Z}$ to mean $\mathbf{Y}-\mathbf{Z}>(\geq) 0$.

Lemma 6. For any matrices $\mathbf{Y}$ and $\mathbf{Z}$,

$$
\begin{aligned}
& \pm \mathbf{Y}^{\top} \mathbf{Z} \pm \mathbf{Z}^{\top} \mathbf{Y} \leq \mathbf{Y}^{\top} \mathbf{Y}+\mathbf{Z}^{\top} \mathbf{Z} \\
& \mp \mathbf{Y}^{\top} \mathbf{Z} \mp \mathbf{Z}^{\top} \mathbf{Y} \geq-\mathbf{Y}^{\top} \mathbf{Y}-\mathbf{Z}^{\top} \mathbf{Z} .
\end{aligned}
$$

Proof. It is trivial from

$$
\mp \mathbf{Y}^{\top} \mathbf{Z} \mp \mathbf{Z}^{\top} \mathbf{Y}+\mathbf{Y}^{\top} \mathbf{Y}+\mathbf{Z}^{\top} \mathbf{Z}=(\mathbf{Y} \mp \mathbf{Z})^{\top}(\mathbf{Y} \mp \mathbf{Z}) \geq 0
$$

\section{Lemma 7.}

$$
\begin{aligned}
{\left[\begin{array}{ll}
\mathbf{Q} & \mathbf{S} \\
\mathbf{S}^{\top} & \mathbf{R}
\end{array}\right]>0 } & \Leftrightarrow \mathbf{R}>0 \text { and } \mathbf{Q}-\mathbf{S R}^{-1} \mathbf{S}^{\top}>0 \\
& \Leftrightarrow \mathbf{Q}>0 \text { and } \mathbf{R}-\mathbf{S}^{\top} \mathbf{Q}^{-1} \mathbf{S}>0 .
\end{aligned}
$$

Proof.

$$
\begin{aligned}
{\left[\begin{array}{ll}
\mathbf{Q} & \mathbf{S} \\
\mathbf{S}^{\top} & \mathbf{R}
\end{array}\right] } & =\left[\begin{array}{cc}
\mathbf{I} & \mathbf{S} \mathbf{R}^{-1} \\
\mathbf{0} & \mathbf{I}
\end{array}\right]\left[\begin{array}{ccc}
\mathbf{Q}-\mathbf{S} \mathbf{R}^{-1} \mathbf{S}^{\top} & \mathbf{0} \\
\mathbf{0} & \mathbf{R}
\end{array}\right]\left[\begin{array}{cc}
\mathbf{I} & \mathbf{0} \\
\mathbf{R}^{-1} \mathbf{S}^{\top} & \mathbf{I}
\end{array}\right] \\
& =\left[\begin{array}{cc}
\mathbf{I} & \mathbf{0} \\
\mathbf{S}^{\top} \mathbf{Q}^{-1} & \mathbf{I}
\end{array}\right]\left[\begin{array}{cc}
\mathbf{Q} & \mathbf{0} \\
\mathbf{0} & \mathbf{R}-\mathbf{S}^{\top} \mathbf{Q}^{-1} \mathbf{S}
\end{array}\right]\left[\begin{array}{cc}
\mathbf{I} & \mathbf{Q}^{-1} \mathbf{S} \\
\mathbf{0} & \mathbf{I}
\end{array}\right]
\end{aligned}
$$

For square matrices $\mathbf{A}$ and $\mathbf{B}$, we denote a block-diagonal matrix as

$$
\mathbf{A} \oplus \mathbf{B} \triangleq\left[\begin{array}{ll}
\mathbf{A} & \mathbf{0} \\
\mathbf{0} & \mathbf{B}
\end{array}\right] .
$$




\section{Appendix B. Proof of Lemma 3}

The solutions of (53) and (54) are

$$
\begin{aligned}
\hat{\omega}_{r \mathrm{opt}}(t) & =e^{-k_{3} t} \hat{\omega}_{\text {ropt }}(0)+k_{3} \int_{0}^{t} e^{-k_{3}(t-\tau)} \omega_{r}(\tau) d \tau, \\
\hat{k}_{\mathrm{opt}}(t) & =e^{-k_{4} t} \hat{k}_{\mathrm{opt}}(0) \\
& +\int_{0}^{t}\left(-\omega_{r}(\tau)^{2} \hat{\omega}_{r \mathrm{opt}}(\tau)+\omega_{r}(\tau)^{3}+k_{4} k_{\mathrm{opt}}^{\prime}\right) e^{-\left(k_{4}-\tau\right)} d \tau .
\end{aligned}
$$

Because $\omega_{r}(t)<\omega_{\text {rrated }}$, we have

$$
\begin{aligned}
\left|\hat{\omega}_{\text {ropt }}(t)\right| & \leq\left|e^{-k_{3} t}\right|\left|\hat{\omega}_{\text {ropt }}(0)\right|+k_{3}\left|\int_{0}^{t} e^{-k_{3}(t-\tau)} \omega_{r}(\tau) d \tau\right| \\
& \leq\left|e^{-k_{3} t}\right|\left|\hat{\omega}_{\text {ropt }}(0)\right|+k_{3} \omega_{\text {rrated }}\left|\int_{0}^{t} e^{-k_{3}(t-\tau)} d \tau\right| \\
& \leq\left|\hat{\omega}_{\text {ropt }}(0)\right|+\left(1-e^{-k_{3} t}\right) \omega_{\text {rrated }} \\
& \leq\left|\hat{\omega}_{\text {ropt }}(0)\right|+\omega_{\text {rrated }}
\end{aligned}
$$

and

$$
\begin{aligned}
\left|\hat{k}_{\mathrm{opt}}(t)\right| \leq & \left|e^{-k_{4} t}\right|\left|\hat{k}_{\mathrm{opt}}(0)\right|+\int_{0}^{t}\left|\omega_{r}(\tau)^{2}\right|\left|\hat{\omega}_{\text {ropt }}(\tau)\right| e^{-k_{4}(t-\tau)} d \tau \\
& +\int_{0}^{t}\left(\left|\omega_{r}(\tau)^{3}\right|+k_{4} k_{\mathrm{opt}}^{\prime}\right) e^{-k_{4}(t-\tau)} d \tau \\
\leq & \left|e^{-k_{4} t}\right|\left|\hat{k}_{\mathrm{opt}}(0)\right| \\
& +\left(\left|\hat{\omega}_{\text {ropt }}(0)\right|+\omega_{\text {rrated }}\right) \int_{0}^{t}\left|\omega_{r}(\tau)^{2}\right| e^{-k_{4}(t-\tau)} d \tau \\
& +\int_{0}^{t}\left(\left|\omega_{r}(\tau)^{3}\right|+k_{4} k_{\mathrm{opt}}^{\prime}\right) e^{-k_{4}(t-\tau)} d \tau \\
\leq & \left|\hat{k}_{\mathrm{opt}}(0)\right|+\left(1-e^{-k_{4} t}\right)\left(k_{4}^{-1} \omega_{\text {rrated }}^{3}+k_{\mathrm{opt}}^{\prime}\right) \\
& +\left(\left|\hat{\omega}_{\text {ropt }}(0)\right|+\omega_{\text {rrated }}\right)\left(1-e^{-k_{4} t}\right) k_{4}^{-1} \omega_{\text {rrated }}^{2} \\
\leq & \left|\hat{k}_{\mathrm{opt}}(0)\right|+k_{4}^{-1} \omega_{\text {rrated }}^{3}+k_{\mathrm{opt}}^{\prime} \\
& +\left(\left|\hat{\omega}_{\text {ropt }}(0)\right|+\omega_{\text {rrated }}\right) k_{4}^{-1} \omega_{\text {rrated }}^{2} \\
\leq & \left|\hat{k}_{\mathrm{opt}}(0)\right|+2 k_{4}^{-1} \omega_{\text {rrated }}^{3}+k_{4}^{-1}\left|\hat{\omega}_{\text {ropt }}(0)\right| \omega_{\text {rrated }}^{2}+k_{\mathrm{opt}}^{\prime} .
\end{aligned}
$$

\section{Appendix C. Proof of Theorem 1}

To use

$$
\mathbf{E}=\left[\begin{array}{cc}
k_{d} & \mathbf{0} \\
\mathbf{0} & \mathbf{I}_{2}
\end{array}\right] \oplus\left[\begin{array}{cc}
\hat{J} & \mathbf{0} \\
\mathbf{0} & \mathbf{I}_{2}
\end{array}\right]>0
$$


and

$$
\mathbf{X}=\left[\begin{array}{cc}
\alpha \mathbf{I}_{3} & \mathbf{0} \\
\mathbf{0} & \mathbf{I}_{3}
\end{array}\right]>0,
$$

we define a Lyapunov function as

$$
V \triangleq \mathbf{e}(t)^{\top} \mathbf{X E} \mathbf{e}(t)
$$

where

$$
\mathbf{e}(t)=\left[\begin{array}{c}
e_{\omega_{\text {ret }}}(t) \\
\mathbf{e}_{i}(t) \\
e_{\omega_{\mathrm{opt}}}(t) \\
e_{\hat{\omega}_{\mathrm{rop}}}(t) \\
e_{k_{\mathrm{opt}}}(t)
\end{array}\right] \triangleq\left[\begin{array}{c}
\omega_{r \mathrm{rref}}(t)-\omega_{r}(t) \\
\mathbf{i}_{\text {rref }}(t)-\mathbf{i}_{r}(t) \\
\omega_{r}(t)-\omega_{\mathrm{ropt}}\left(V_{w}(t)\right) \\
\omega_{r \mathrm{opt}}\left(V_{w}(t)\right)-\hat{\omega}_{r \mathrm{opt}}(t) \\
k_{\mathrm{opt}}-\hat{k}_{\mathrm{opt}}(t)
\end{array}\right]
$$

In this proof, we show that the derivative of the Lyapunov function

$$
\dot{V}=\mathbf{e}(t)^{\top} \mathbf{X E} \frac{\mathrm{d}}{\mathrm{d} t} \mathbf{e}(t)+\left(\mathbf{E} \frac{\mathrm{d}}{\mathrm{d} t} \mathbf{e}(t)\right)^{\top} \mathbf{X} \mathbf{e}(t),
$$

satisfies

$$
\dot{V}<-q V+\epsilon \text {. }
$$

By defining the function $p(t) \triangleq-q V+\epsilon-\dot{V}>0$, we have

$$
\dot{V}=-q V+\epsilon-p(t) .
$$

Then,

$$
\begin{aligned}
V(t) & =e^{-q t} V(0)+\int_{0}^{t} e^{-q(t-\tau)}(\epsilon-p(\tau)) d \tau \\
& <e^{-q t} V(0)+\int_{0}^{t} e^{-q(t-\tau)} \epsilon d \tau \\
& =e^{-q t} V(0)+\frac{\epsilon}{q}\left(1-e^{-q t}\right) .
\end{aligned}
$$

Hence, the upper bound of $V(t)$ converges to $\epsilon / q$. This implies that $V(t)$ will be bounded by $\epsilon / q$ as $t$ increases. Since $\hat{J} e_{\omega_{\text {opt }}}(t)^{2} \leq V(t)$, there exists a time $t_{o}>0$ such that for all $t \geq t_{o}$,

$$
\left|e_{\omega_{\mathrm{opt}}}(t)\right|=\left|\omega_{r}(t)-\omega_{\text {ropt }}\left(V_{w}(t)\right)\right|<\sqrt{\frac{\epsilon}{\hat{J} q}}
$$

is satisfied.

Hereafter, we will derive (C.6). 
From (2), (72), and (51), we have

$$
\begin{aligned}
& J \omega_{r}(t) \frac{\mathrm{d}}{\mathrm{d} t} \omega_{r}(t)=P_{m}(t)-P_{e}(t) \\
& =k_{\mathrm{opt}} \omega_{r}(t)^{3}-\zeta(t) \omega_{r}(t) e_{\omega_{\mathrm{opt}}}(t)-\hat{k}_{\mathrm{opt}}(t) \omega_{r \mathrm{ref}}(t)^{3} \\
& +\omega_{r}(t)\left(k_{1} \frac{\mathrm{d}}{\mathrm{d} t} \omega_{r}(t)-k_{2}\left(\omega_{r}(t)-\hat{\omega}_{r \mathrm{opt}}(t)\right)\right) .
\end{aligned}
$$

Then, when we use $\hat{J}=J-k_{1}$ and $\omega_{\text {ref }}(t)^{3}-\omega_{r}(t)^{3}=\xi\left(\omega_{r}(t), \omega_{\text {rref }}(t)\right) \omega_{r}(t) e_{\omega_{\text {ref }}}(t)$, and $\omega_{r}(t)-\hat{\omega}_{r \text { opt }}(() t)=e_{\omega_{\text {opt }}}(t)+$ $e_{\hat{\omega}_{\text {ropt }}}(t)$, we have

$$
\begin{aligned}
\hat{J} \frac{\mathrm{d}}{\mathrm{d} t} \omega_{r}(t)= & k_{\mathrm{opt}} \omega_{r}(t)^{2}-\zeta(t) e_{\omega_{\mathrm{opt}}}(t)-\hat{k}_{\mathrm{opt}}(t) \omega_{r}(t)^{2} \\
& -\hat{k}_{\mathrm{opt}}(t) \xi\left(\omega_{r}(t), \omega_{r \mathrm{ref}}(t)\right) e_{\omega_{\text {ref }}}(t)-k_{2}\left(\omega_{r}(t)-\hat{\omega}_{\text {ropt }}(t)\right) \\
= & \left(k_{\mathrm{opt}}-\hat{k}_{\mathrm{opt}}(t)\right) \omega_{r}(t)^{2}-\zeta(t) e_{\omega_{\mathrm{opt}}}(t) \\
& -\hat{k}_{\mathrm{opt}}(t) \xi\left(\omega_{r}(t), \omega_{\mathrm{rref}}(t)\right) e_{\omega_{\text {ref }}}(t)-k_{2}\left(e_{\omega_{\mathrm{opt}}}(t)+e_{4}(t)\right) \\
= & -\zeta(t) e_{\omega_{\mathrm{opt}}}(t)-k_{2}\left(e_{\omega_{\mathrm{opt}}}(t)+e_{\hat{\omega}_{\mathrm{ropt}}}(t)\right) \\
& +\omega_{r}(t)^{2} e_{k_{\mathrm{opt}}}(t)-\hat{k}_{\mathrm{opt}}(t) \xi\left(\omega_{r}(t), \omega_{\mathrm{rref}}(t)\right) e_{\omega_{\text {ree }}}(t)
\end{aligned}
$$

Hence,

$$
\begin{aligned}
\hat{J} \frac{\mathrm{d}}{\mathrm{d} t} e_{3}(t)= & \hat{J} \frac{\mathrm{d}}{\mathrm{d} t} \omega_{r}(t)-\hat{J} \frac{\mathrm{d}}{\mathrm{d} t} \omega_{r \mathrm{opt}}\left(V_{w}(t)\right) \\
= & -\left(\zeta(t)+k_{2}\right) e_{\omega_{\mathrm{opt}}}(t)-k_{2} e_{\hat{\omega}_{\text {ropt }}}(t)+\omega_{r}(t)^{2} e_{k_{\mathrm{opt}}}(t) \\
& -\hat{k}_{\mathrm{opt}}(t) \xi\left(\omega_{r}(t), \omega_{\text {ref }}(t)\right) e_{\omega_{\text {ref }}}(t)-\hat{J} \frac{\mathrm{d}}{\mathrm{d} t} \omega_{r \mathrm{opt}}\left(V_{w}(t)\right) .
\end{aligned}
$$

From [33, 50, 54, 53, , and (C.11), we can summarize

$$
\begin{aligned}
\mathbf{E} \frac{\mathrm{d}}{\mathrm{d} t} \mathbf{e}(t)= & \mathbf{A}(t) \mathbf{e}(t)+\mathbf{B} \frac{\mathrm{d}}{\mathrm{d} t} \omega_{r \mathrm{ropt}}(t)+\mathbf{C} \delta \\
& +\mathbf{D} \hat{k}_{\mathrm{opt}}(t) \xi\left(\omega_{r}(t), \omega_{r \mathrm{ref}}(t)\right) \mathbf{G e}(t),
\end{aligned}
$$


where

$$
\begin{aligned}
& \mathbf{A}(t)=\left[\begin{array}{cc}
\mathbf{A}_{1} & \mathbf{0} \\
\mathbf{0} & \mathbf{A}_{2}(t)
\end{array}\right], \mathbf{B}=\left[\begin{array}{c}
0 \\
0 \\
-\hat{J} \\
1 \\
0
\end{array}\right], \mathbf{C}=\left[\begin{array}{c}
0 \\
0 \\
0 \\
0 \\
-k_{4}
\end{array}\right], \mathbf{D}=\left[\begin{array}{c}
0 \\
0 \\
-1 \\
0 \\
0
\end{array}\right], \\
& \mathbf{G}=\left[\begin{array}{lllll}
1 & 0 & 0 & 0 & 0
\end{array}\right], \\
& \mathbf{A}_{1}=-\left[\begin{array}{cc}
k_{p} & {\left[\begin{array}{cc}
0 & -1
\end{array}\right]} \\
\mathbf{0} & \mathbf{K}
\end{array}\right] \text {, } \\
& \mathbf{A}_{2}(t)=\left[\begin{array}{ccc}
-\left(\zeta(t)+k_{2}\right) & -k_{2} & J \omega_{r}(t)^{2} \\
-k_{3} & -k_{3} & 0 \\
-\omega_{r}(t)^{2} & -\omega_{r}(t)^{2} & -k_{4}
\end{array}\right] .
\end{aligned}
$$

By substituting $(\overline{C .12})$ into $(\overline{C .5})$, we have

$$
\begin{aligned}
\dot{V}= & \mathbf{e}(t)^{\top}\left(\mathbf{X A}(t)+\mathbf{A}(t)^{\top} \mathbf{X}\right) \mathbf{e}(t)+2 \mathbf{e}(t)^{\top} \mathbf{X B} \frac{\mathrm{d}}{\mathrm{d} t} \omega_{r \mathrm{opt}}(t) \\
& +2 \mathbf{e}(t)^{\top} \mathbf{C} \delta+2 \mathbf{e}(t)^{\top} \mathbf{X} \mathbf{D} \hat{k}_{\mathrm{opt}}(t) \xi\left(\omega_{r}(t), \omega_{\text {ref }}(t)\right) \mathbf{G e}(t) .
\end{aligned}
$$

Since $2\left|e_{\omega_{\text {opt }}}(t)\right| \leq w e_{\omega_{\text {opt }}}(t)^{2}+w^{-1}$ and $2\left|e_{\hat{\omega}_{\text {ropt }}}(t)\right| \leq w e_{\hat{\omega}_{\text {ropt }}}(t)^{2}+w^{-1}$ for $w>0$, we have

$$
\begin{aligned}
2 \mathbf{e}(t)^{\top} \mathbf{X B} \frac{\mathrm{d}}{\mathrm{d} t} \omega_{r \text { opt }}(t) & \leq 2\left|-\hat{J} e_{\omega_{\text {opt }}}(t)+e_{\hat{\omega}_{\text {ropt }}}(t)\right|\left|\frac{\mathrm{d}}{\mathrm{d} t} \omega_{\text {ropt }}\left(V_{w}(t)\right)\right| \\
& \leq 2\left(\hat{J}\left|e_{\omega_{\text {opt }}}(t)\right|+\left|e_{\hat{\omega}_{\text {ropt }}}(t)\right|\right) \gamma \\
& \leq\left(w \hat{J} e_{\omega_{\text {opt }}}(t)^{2}+w e_{\hat{\omega}_{\text {ropt }}}(t)^{2}+w^{-1} \hat{J}+w^{-1}\right) \gamma \\
& =\mathbf{e}(t)^{\top} \mathbf{M e}(t)+\epsilon_{1},
\end{aligned}
$$

where

$$
\begin{aligned}
& \mathbf{M} \triangleq w \gamma \operatorname{diag}(0,0, \hat{J}, 1,0), \\
& \epsilon_{1} \triangleq w^{-1} \gamma(\hat{J}+1) .
\end{aligned}
$$

Likely, we have

$$
2 \mathbf{e}(t)^{\top} \mathbf{X C} \delta<2 k_{4}\left|e_{k_{\mathrm{opt}}}(t)\right|\left|\delta_{\max }\right| k_{\mathrm{opt}} \leq \mathbf{e}(t)^{\top} \mathbf{N e}(t)+\epsilon_{2},
$$

where $\mathbf{N}=\operatorname{diag}\left(0,0,0,0, v k_{\mathrm{opt}} k_{4}\right), \epsilon_{2}=v^{-1} k_{4} k_{\mathrm{opt}} \delta_{\max }^{2}$. Furthermore, by applying A.4 in Lemma 6 to set $\mathbf{Y}=$ 
$\alpha \hat{k}_{\mathrm{opt}}(t) \xi\left(\omega_{r}(t), \omega_{\text {rref }}(t)\right) \mathbf{G}$ and $\mathbf{Z}=\alpha^{-1} \mathbf{D}^{\top} \mathbf{X}$, we have

$$
\begin{aligned}
2 \mathbf{e}(t)^{\top} \mathbf{X D} \hat{k}_{\mathrm{opt}}(t) \xi\left(\omega_{r}(t), \omega_{\text {ref }}(t)\right) \mathbf{G e}(t) \\
=\mathbf{e}(t)^{\top} \mathbf{G}^{\top} \hat{k}_{\mathrm{opt}}(t) \xi\left(\omega_{r}(t), \omega_{\mathrm{rref}}(t)\right) \mathbf{D}^{\top} \mathbf{X e}(t) \\
\quad+\mathbf{e}(t)^{\top} \mathbf{X D} \hat{k}_{\mathrm{opt}}(t) \xi\left(\omega_{r}(t), \omega_{\text {rref }}(t)\right) \mathbf{G e}(t) \\
\leq \mathbf{e}(t)^{\top} \alpha^{2} \hat{k}_{\mathrm{opt}}(t)^{2} \xi\left(\omega_{r}(t), \omega_{\text {ref }}(t)\right)^{2} \mathbf{G}^{\top} \mathbf{G e}(t) \\
\quad+\alpha^{-2} \mathbf{e}(t)^{\top}(t) \mathbf{X D D}^{\top} \mathbf{X e}(t) .
\end{aligned}
$$

Then, by noting that $\alpha^{-2} \mathbf{X D D}^{\top} \mathbf{X}=\mathbf{D D}^{\top}$ and defining

$$
\begin{aligned}
\mathbf{Q}(t) \triangleq & -\mathbf{X A}(t)-\mathbf{A}(t)^{\top} \mathbf{X}-\mathbf{M}-\mathbf{N}-\mathbf{D D}^{\top} \\
& -\alpha^{2} \hat{k}_{\mathrm{opt}}(t)^{2} \xi\left(\omega_{r}(t), \omega_{\mathrm{rref}}(t)\right)^{2} \mathbf{G}^{\top} \mathbf{G}, \\
\epsilon \triangleq & \epsilon_{1}+\epsilon_{2},
\end{aligned}
$$

we have

$$
\dot{V} \leq-\mathbf{e}(t)^{\top} \mathbf{Q}(t) \mathbf{e}(t)+\epsilon
$$

Hence, if

$$
\mathbf{Q}(t)-q \mathbf{X E}>0,
$$

then

$$
\dot{V}<-q \mathbf{e}(t)^{\top} \mathbf{X E e}(t)+\epsilon
$$

This implies C.6. To complete the proof, we need the next lemma.

Lemma 8. The five inequalities in 59) imply C.25.

Proof. $q \mathbf{X E}$ and $\mathbf{Q}(t)$ are both block diagonal as

$$
\begin{aligned}
q \mathbf{X E} & =q \alpha\left[\begin{array}{ll}
k_{d} & \mathbf{0} \\
\mathbf{0} & \mathbf{I}_{2}
\end{array}\right] \oplus q\left[\begin{array}{ll}
\hat{J} & \mathbf{0} \\
\mathbf{0} & \mathbf{I}_{2}
\end{array}\right], \\
\mathbf{Q}(t) & =\alpha\left[\begin{array}{cc}
2 k_{p}-\alpha \hat{k}_{\mathrm{opt}}(t)^{2} \xi\left(\omega_{r}(t), \omega_{r \mathrm{ref}}(t)\right)^{2} & {\left[\begin{array}{cc}
0 & -1
\end{array}\right]} \\
{\left[\begin{array}{c}
0 \\
-1
\end{array}\right]} & \mathbf{K}^{\top}+\mathbf{K}
\end{array}\right] \\
& \oplus\left[\begin{array}{ccc}
2 \zeta(t)+2 k_{2}-w \gamma \hat{J}-1 & k_{2}+k_{3} & 0 \\
k_{2}+k_{3} & 2 k_{3}-w \gamma & \omega_{r}(t)^{2} \\
0 & \omega_{r}(t)^{2} & \left(2-v k_{\mathrm{opt}}\right) k_{4}
\end{array}\right] .
\end{aligned}
$$


Hence, we show (C.25) separately as

$$
\begin{aligned}
& {\left[\begin{array}{cc}
2 k_{p}-\alpha \hat{k}_{\mathrm{opt}}(t)^{2} \xi\left(\omega_{r}(t), \omega_{r \mathrm{ref}}(t)\right)^{2} & {\left[\begin{array}{ll}
0 & -1
\end{array}\right]} \\
{\left[\begin{array}{c}
0 \\
-1
\end{array}\right]} & \mathbf{K}^{\top}+\mathbf{K}
\end{array}\right]-q\left[\begin{array}{cc}
k_{d} & \mathbf{0} \\
\mathbf{0} & \mathbf{I}_{2}
\end{array}\right]>0,} \\
& {\left[\begin{array}{ccc}
2 \zeta(t)+2 k_{2}-w \gamma \hat{J}-1 & k_{2}+k_{3} & 0 \\
k_{2}+k_{3} & 2 k_{3}-w \gamma & \omega_{r}(t)^{2} \\
0 & \omega_{r}(t)^{2} & \left(2-v k_{\mathrm{opt}}\right) k_{4}
\end{array}\right]-q\left[\begin{array}{ll}
\hat{J} & \mathbf{0} \\
\mathbf{0} & \mathbf{I}_{2}
\end{array}\right]>0 .}
\end{aligned}
$$

By noting that $\hat{k}_{\mathrm{opt}, \mathrm{ub}} \xi_{\max } \geq \hat{k}_{\mathrm{opt}}(t) \xi\left(\omega_{r}(t), \omega_{\text {ref }}(t)\right)$ and applying 47 , the first two inequalities in 59, imply (C.29).

$$
\left\{\begin{array}{l}
2 k_{p}-\alpha \hat{k}_{\mathrm{opt}, \mathrm{ub}}^{2} \xi_{\max }^{2}-\left[\begin{array}{ll}
0 & -1
\end{array}\right] \boldsymbol{\Xi}^{-1}\left[\begin{array}{c}
0 \\
-1
\end{array}\right]-q k_{d}>0, \\
\boldsymbol{\Xi}=\mathbf{K}^{\top}+\mathbf{K}-q \mathbf{I}_{2}>0 .
\end{array}\right.
$$

To apply (A.5) in Lemma 6 by setting

$$
\mathbf{Y}=\left[\begin{array}{ccc}
\sqrt{k_{2}+k_{3}} & 0 & 0 \\
0 & 0 & \omega_{r}(t)
\end{array}\right], \mathbf{Z}=\left[\begin{array}{ccc}
0 & \sqrt{k_{2}+k_{3}} & 0 \\
0 & \omega_{r}(t) & 0
\end{array}\right],
$$

the off-diagonal elements of C.30 are bounded as

$$
\begin{aligned}
& {\left[\begin{array}{ccc}
0 & k_{2}+k_{3} & 0 \\
k_{2}+k_{3} & 0 & \omega_{r}(t)^{2} \\
0 & \omega_{r}(t)^{2} & 0
\end{array}\right]} \\
& \geq-\left[\begin{array}{ccc}
k_{2}+k_{3} & 0 & 0 \\
0 & k_{2}+k_{3}+\omega_{r}(t)^{2} & 0 \\
0 & 0 & \omega_{r}(t)^{2}
\end{array}\right] .
\end{aligned}
$$

By noting that $\zeta(t) \geq \zeta_{\min }$ and $\omega_{r}(t) \leq \omega_{\text {rated }}$, the last three inequalities in 59] imply [C.30.

$$
\left\{\begin{array}{l}
2 \zeta_{\min }-w \hat{J} \gamma-\left(k_{3}-k_{2}\right)-1>q \hat{J} \\
k_{3}-k_{2}-\omega_{\text {rated }}^{2}-w \gamma>q \\
\left(2-v k_{\text {opt }}\right) k_{4}-\omega_{\text {rrated }}^{2}>q .
\end{array}\right.
$$

This completes the proof.

[1] Phan DC, Yamamoto S. Maximum Energy Output of a DFIG Wind Turbine Using an Improved MPPT-Curve Method. Energies 2015; 8: $11718-11736$

[2] Xie D, Xu Z, Yang L, Ostergaard J, Xue Y, Wong KP. A comprehensive LVRT control strategy for DFIG wind turbines with enhanced reactive power support. IEEE Trans. Power Syst. 2013; 28(3): 3302-3310. 
[3] Alizadeh M, Kojori SS. Augmenting effectiveness of control loops of a PMSG (permanent magnet synchronous generator) based wind energy conversion system by a virtually adaptive PI (proportional integral) controller. Energy 2015; 91: 610-629.

[4] Ganjefar S, Ghasemi AA. A novel-strategy controller design for maximum power extraction in stand-alone windmill systems. Energy 2014; 76: $326-335$.

[5] Barakati SM, Kazerani M, Aplevich JD. Maximum power tracking control for a wind turbine system including a matrix converter. IEEE Trans. Energy Convers. 2009; 24(3): 705-713.

[6] Lei Y, Mullane A, Lightbody G, Yacamini R. Modeling of the wind turbine with a doubly fed induction generator for grid integration studies. IEEE Trans. Energy Convers. 2006; 21(1): 257-264.

[7] Abdullah M, Yatim AHM, Tan C, Saidur R. A review of maximum power point tracking algorithms for wind energy systems. Renew. Sustain. Energy Rev. 2012; 16(5): 3220-3227.

[8] Ganjefar S, Ghassemi A, Ahmadi M. Improving efficiency of two-type maximum power point tracking methods of tip-speed ratio and optimum torque in wind turbine system using a quantum neural network. Energy 2014; 67: 444-453.

[9] Jeong HG, Seung RH, Lee KB. An improved maximum power point tracking method for wind power systems. Energies 2012; 5: $1339-1354$.

[10] Phan DC, Ro KS. Point of common coupling voltage recovery capability improvement of offshore wind farm connected to a weak grid through high voltage direct current link. J. Renew. Sustain. Energy 2012; 4: 063109(1-13).

[11] Tapia A, Tapia G, Ostolaza J, Saenz J. Modeling and control of a wind turbine driven doubly fed induction generator. IEEE Trans. Energy Convers. 2003; 18(2): 194-204.

[12] Fernandez L, Garcia C, Jurado F. Comparative study on the performance of control systems for doubly fed induction generator (DFIG) wind turbines operating with power regulation. Energy 2008; 33(9): 1438-1452.

[13] Yang L, Xu Z, stergaard J, Dong ZY, Wong KP. Advanced control strategy of DFIG wind turbines for power system fault ride through. IEEE Trans. Power Syst. 2012; 27(2): 713-722.

[14] Wagner H, Mathur J. Introduction to wind energy systems: Basics, technology and operation. 2nd ed. Operation and control of wind energy converters, New York: Springer-Verlag; 2013, p. 63-64.

[15] Belmokhtar K, Doumbia ML, Agbossou K. Novel fuzzy logic based sensorless maximum power point tracking strategy for wind turbine systems driven DFIG (doubly-fed induction generator). Energy 2014; 76: 679-693.

[16] Yang L, Xu Z, stergaard J, Dong ZY, Wong KP, Ma X. Oscillatory stability and eigenvalue sensitivity analysis of a DFIG wind turbine system. IEEE Trans. Energy Convers. 2011; 26(1): 328-339.

[17] Mishra Y, Mishra S, Li F, Dong ZY, Bansal RC. Small signal stability analysis of a DFIG-based wind power system under different modes of operation. IEEE Trans. Energy Convers. 2009; 24(4): 972-982.

[18] Hu J, Nian H, Hu B, He Y, Zhu ZQ. Direct active and reactive power regulation of DFIG using sliding-mode control approach. IEEE Trans. Energy Convers.2010; 25(4): 1028-1039.

[19] Khemiri N, Khedher A, Mimouni MF. Wind energy conversion system using DFIG controlled by backstepping and sliding mode strategies. Int. J. Renew. Energy Res. 2012; 2(3): 421-430.

[20] Lin W, Hong C, Cheng F. On-line designed hybrid controller with adaptive observer for variable-speed wind generation system. Energy 2010; 35: 3022-3030.

[21] Evangelista C, Puleston P, Valenciaga F, Fridman LM. Lyapunov-designed super-twisting sliding mode control for wind energy conversion optimization. IEEE Trans. Ind. Electron. 2013; 60(2): 438-454.

[22] Barambones O, Durana JMG, Sen MD. Robust speed control for variable speed wind turbine. Int. J. Innov. Comput. Inform. Contr. 2012; 8(11): 7627-7640.

[23] Beltran B, Ahmed-Ali T, Benbouzid M. High-order sliding-mode control of variable-speed wind turbines. IEEE Trans. Ind. Electron. 2009; 56(9): 3314-3321.

[24] Wu B, Lang Y, Zargari N, Kouro S. Power conversion and control of wind energy system. Hoboken, New Jersey: John Wiley \& Sons; 2011.

[25] Abad G, López J, Rodríguez MA, Marroyo L, Iwanski G. Doubly fed induction machine: Modelling and control for wind energy generation. 
Hoboken, New Jersey: John Wiley \& Sons; 2011.

[26] González LG, Figueres E, Garcerá G, Carranza O. Maximum-power-point tracking with reduced mechanical stress applied to wind-energyconversion-systems. Appl. Energy 2010; 87: 2304-2312.

[27] Ghaffari A, Krstic M, Seshagiri S. Power optimization and control in wind energy conversion systems using extremum seeking. IEEE Trans. Control Syst. Tech. 2014; 22(5): 1684-1695.

[28] Qiao W, Yang X, Gong X. Wind speed and rotor position sensorless control for direct-drive PMG wind turbines. IEEE Trans. Ind. Appl. 2012; 48(1): 3-11.

[29] Qiao W, Zhou W, Aller JM, Harley RG. Wind speed estimation based sensorless output maximization control for a wind turbine driving a DFIG. IEEE Trans. Power. Electron. 2008; 23(3): 1156-1169.

[30] Boyd S, Ghaoui L. El, Feron E, Balakrishnan V. Linear matrix inequalities in system and control theory. Philadelphia: SIAM; 1994.

[31] Boyd S, Vandenberghe L. Convex optimization. New York: Cambridge University Press; 2004. 